Bangladesh J. Plant Taxon. 23(1): 13-25, 2016 (June)

(C) 2016 Bangladesh Association of Plant Taxonomists

\title{
AN ENUMERATION TO THE ORCHIDS AND THEIR CONSERVATION STATUS IN GREATER SYLHET, BANGLADESH
}

\author{
M.M. ISLAM, M.K. HUdA ${ }^{1}$ AND M. HALIM ${ }^{2}$ \\ Department of Botany, University of Chittagong, Chittagong 4331, Bangladesh
}

Keywords: Conservation; Diversity; Orchidaceae; Sylhet.

\begin{abstract}
The present investigation deals with enumeration including diversity, ecology and conservation of the family Orchidaceae of greater Sylhet region of Bangladesh. Extensive field trips were made at 11 different sites of this region during early monsoon, late monsoon and winter seasons. Relevant literature and different herbaria were consulted to gather information about the orchids of this region. Orchidaceae is represented in greater Sylhet by 75 species under 49 genera. Out of these, 25 species are terrestrial, 48 are epiphytic, one is saprohytic and one is hemiepiphytic. Presence of 37 monotypic genera indicates a narrow diversity in Orchidaceae of this area. The present investigation revealed that 26 orchid species are restricted and distributed only in Sylhet region in Bangladesh. The currently accepted taxonomic nomenclature, synonyms, habit, flowering time, present conservation status and geographical distribution are provided under each taxon.
\end{abstract}

\section{Introduction}

Orchidaceae is one of the largest flowering plant families, represented by about 1000 genera and 20,000 species with cosmopolitan distribution, primarily in the tropics and rarely in arctic regions (Chowdhery, 1998). A preliminary checklist of family Orchidaceae for Bangladesh was made by Huda et al. (1999) with an enumeration of 160 species and 2 varieties under 63 genera for Bangladesh. Of them, 106 taxa were epiphytic and remaining 56 were terrestrial. Huda et al. (2001) added some new records for the family of Orchidaceae from Bangladesh. The distribution of terrestrial orchids in Bangladesh was compiled as a check list mainly on the basis of previous records, literature survey and herbarium collections (Khanam et al., 2001).

Diversity and ecology of the orchids in the south-eastern part of Bangladesh have also been studied by Huda (2000). Earlier reports indicate that Sylhet region was rich in orchid diversity (Hooker 1890a, b; Prain, 1903). Some research works on orchids from Bangladesh were done sporadically, viz. Huda et al. (1999), Huda (2000, 2008, 2008a), Ahmed and Pasha (1993, 1993a, 1994, 1998, 1998a, 1998b, 1999) as part of their floral exploration but focus has not been given to the diversity and ecology of orchids of Sylhet region. Valuable herbarium specimens of orchids from the greater Sylhet regions, collected by many taxonomists of Bangladesh are housed at the different herbaria of Bangladesh, viz. Bangladesh National Herbarium (DACB), Dhaka University Salar Khan Herbarium (DUSH), Herbarium of Chittagong University (HCU), Herbarium of Bangladesh Forest Research Institute (HBFRI) and Herbarium of BCSIR laboratory (HBCSIR). The present study was, therefore, undertaken with a view to examine the previous specimens and relevant literatures, and also to conduct field investigation for collecting specimens of the family occurring in the area, particularly in the greater Sylhet region mainly in the forest areas of Sylhet,

${ }^{1}$ Corresponding author. Email: mkhuda70@hotmail.com

${ }^{2}$ Chittagong Education Board, Chittagong, Bangladesh. 
Moulavi Bazar, Sunamganj and Habiganj of Bangladesh for a taxonomic treatment of the family Orchidaceae.

\section{Materials and Methods}

Study area

The floristic study of the family Orchidaceae was conducted from July 2006 to January 2010 in Lawachara National Park, Madhabkunda Eco-Park, Bangladesh Tea Research Institute (BTRI) Campus in Moulvi Bazar; Rema-Kalenga Wildlife Sanctuary and Satchari National Park in Habiganj; Jaflong, Tamabil, Sripur, Jaintapur, Tilagarh forest beat in Sylhet district and Sadar of Sunamganj district. Eleven different sites in four districts of greater Sylhet region were visited to study the diversity and ecology of orchids.

\section{Collection of specimens}

Orchid specimens with detailed information were collected both in the flowering and nonflowering stage from the study area through eight field trips each consisting of 4 to 5 days in early monsoon (March to May), monsoon (June to July), late monsoon (August to October) and winter (November to February).

Herbarium and literature survey

Both living and herbarium specimens were examined and studied carefully at the Herbarium of Chittagong University (HCU). Herbarium specimens of orchids collected in the present study were studied and matched with herbarium specimens available at DACB, DUSH, HBFRI, HBCSIR, and HCU. Local orchid experts were consulted to identify some specimens and to confirm some critical specimens. In order to compare the description, nomenclature and geographical distribution and uses, Roxburgh $(1814,1832)$ Hooker (1890a, b), Prain (1903), Heinig (1925), Bruhl (1926), Sinclair (1956), Abraham and Vatsala (1981), Joseph (1987) and Huda (2000) were consulted.

Abundance status was measured based on observation, availability of the species in the field, herbarium specimen preserved at DACB, DUSH, HCU and HBFRI and literature survey following Misra (2000) and Rao (1998). Categories for Abundance status based on their availability are mentioned as Specimen deficient (no collection or herbarium specimen is available in any herbarium of Bangladesh), Rare (only one herbarium specimen found but no further collection made after record), Scarce (one or two herbarium specimens available and collected once or twice after record), Occasional (few herbarium specimens available and collected from one or few localities from other parts of Bangladesh also), and Common (usually occur in the different areas of greater Sylhet and other parts of Bangladesh).

\section{Taxonomic enumeration to the species}

The present study identified 75 species belonging to 49 genera of Orchidaceae in the greater Sylhet region. Enumeration is presented below alphabetically. An asterisk $(*)$ at the beginning of the species name indicates its occurrence from the greater Sylhet region only, on the other hand, another asterisk $\left(^{*}\right)$ used at the end of synonym in the enumeration to indicate that it is the first recorded name, if applicable. Flowering time ( $\mathrm{Fl}$.) of the species is presented numerically from 1 to 12 for January to December, respectively. Categories of Abundance status follows the flowering time.

1. Acampe papillosa (Lindl.) Lindl., Fol. Orchid. 2 (1853). Saccolabium papillosum Lindl. (1841); Gastrochilus papillosus (Lindl.) O. Kuntze (1891). Reported by its synonym from Chittagong and the Sundarbans by Prain (1903). Epiphytic. Fl.: 8-9. Common. Distribution: India 
and Bangladesh (Chittagong, Cox's Bazar, Bandarban, Rangamati, Khagrachari and greater Sylhet). Specimen examined: Habiganj: Rema-Kalenga; 25.02.2007, M. Islam 01 (HCU).

2. *Acanthephippium sylhetense Lindl., Gen. Sp. Orchid. Pl.: 177 (1833). Reported from Sylhet by Hooker (1890a). Terrestrial. Fl.: 4-5. Specimen deficient. Distribution: China, Fiji Island, India and Bangladesh (Sylhet).

3. Aerides crispa Lindl., Gen. Sp. Orchid. : 239 (1833). Aerides lindleyana Wight, Ic. t. 1677. (1851). Reported by Ahmed et al. (1989). Epiphytic. Fl.: 3-5. Rare. Distribution: Southern India to Myanmar, Bangladesh (Chittagong and Sylhet). Specimen examined: Sylhet: Lama Bazar; 21.3.1988, M. Ahmed 122 (HCU).

4. Aerides multiflorum Roxb., Pl. Cor. 3: 68, t. 271 (1820). Aerides affine Lindl. (1833); Aerides multiflora var. dactyloides Mokter et al. (1989). Roxburgh (1832) reported from Sylhet. Epiphytic. Fl.: 5-6. Occasional. Distribution: India, Malaysia, Philippines, Thailand and Bangladesh (Cox's Bazar, Rangamati and Sylhet). Specimen examined: Sylhet: Lama Bazar (Near college road); 21.03. 1986, M. Ahmed, 97 (HCU).

5. Aerides odoratum Lour., Fl. Cochinch. 2: 525 (1790). Aerides cornutum Roxb. (1832). Reported by its synonym from Dhaka by Roxburgh (1832). Epiphytic. Fl.: 5-6. Common. Distribution: China, India, Malaysia, Myanmar, Nepal and Bangladesh (distributed in most of the areas of south-east part of Bangladesh and greater part of Sylhet). Specimens examined: Sylhet: Jainta bazar; 10.10.81; Moyeen 70 (HCU); Lawachara; 27. 02. 07, M. Islam and M. K. Huda 11 ( $\mathrm{HCU})$.

6. *Aerides suavissima Lindl. N. Journ. Hort. Soc. Iv. : 263 (1858). Hooker, f. (1890b) reported from Sylhet. Epiphytic. Fl.: 5-6. Rare. Distribution: Malaysia, Myanmar and Bangladesh (Sylhet). Specimen examined: Sylhet: Tamabil; 23.03.86, M. Ahmed 130 (HCU).

7. Agrostophyllum khasianum Griff. Calcutta J. Nat. Hist. 4: 376, t. 19. (1844). Appendicula hasseltii Wight. (1851). Reported from Sylhet by Ahmed et al. (1989a). Epiphytic. Fl.: 4-5. Scarce. Distribution: India and Bangladesh (Cox' Bazar and Sylhet). Specimen examined: Sunamganj: Bagan bari, Sadar; 25.4.1986, M. Ahmed 116 (HCU).

8. *Anaectochilus roxburghii (Wall.) Lindl., Gen. Sp. Orchid. Pl.: 499 (1840). Chrysobaphus roxburghii Wall. (1826); Anaectochilus yungianus Hu (1971); Zeuxine roxburghii (Lindl.) Hiroe (1971). Lindley (1830-40) reported it from Sylhet. Terrestrial. Fl.: 4-6. Specimen deficient. Distribution: Bhutan, China, India, Laos, Thailand, Vietnam and Bangladesh (Sylhet).

9. Arundina graminifolia (D. Don) Hochr. in Bull. New York Bot. Gard. 6: 270 (1910). Blettia graminifolia D. Don (1825); Limodorum graminifolia Buch.-Ham. ex D. Don (1825); Arundina bambusifolia Lindl. (1830); Cymbidium bambusifolium Roxb. (1832). Reported from Chittagong by Roxburgh (1814, 1832). Terrestrial. Fl.: 12-3. Occasional. Distribution: China, India, Malaysia, Myanmar, Philippines, Sri Lanka and Bangladesh (Bandarban, Chittagong, Cox's Bazar, Khagrachari, Rangamati and Sylhet). Specimen examined: Dhaka: 07.09. 46, S. K. Sen (DUSH).

10. Brachycorythis helferi (Rchb. f.) Summerh. in Kew Bull. 1955: 235 (1955). Gymnadenia helferi Rchb. f. (1872); Habenaria helferi (Rchb. f.) Hook. f. (1890b). Terrestrial. Fl.: 8-9. Scarce. Distribution: India, Myanmar and Bangladesh (Bandarban and Sylhet). Uddin et al. (2000) reported it from Habiganj. Specimen examined: Bandarban: Chimbuk hills, 08. 09. 99, M.A. Rahman et al. 5744c (HCU). 
11. Bulbophyllum lilacinum Ridl. in J. Linn. Soc. 32: 276 (1896). Epiphytic. Fl.: 10-11. Common. Distribution: India, Malaya Peninsula and Bangladesh throughout Cox's Bazar district (Khan and Halim, 1987) and Sylhet. Specimen examined: Sylhet: Tamabil, 12. 06. 07, M. Islam 02 (HCU).

12. *Calanthe densiflora Lindl., Gen. Sp. Orchid. Pl.: 250 (1833). Alimorchis densiflora (Lindl.) Kuntze (1891); Calanthe kazuoi Yamamoto (1930). Reported from Sylhet by Lindley (1830-40). Terrestrial. Fl.: 10-12. Specimen deficient. Distribution: Bhutan, China, India, Japan, Nepal, Vietnam and Bangladesh (Sylhet).

13. *Calanthe puberula Lindl., Gen. Sp. Orchid. Pl.: 252 (1833). Alismorchis puberula (Lindl.) Kuntze (1891); Calanthe amoena Smith (1921); C. lepida Smith (1921). Lindley (1830-40) reported from Sylhet. Terrestrial. Fl.: 10-12. Specimen deficient. Distribution: Bhutan, China, India, Myanmar, Taiwan, Vietnam and Bangladesh (Sylhet).

14. Cephalantheropsis gracilis (Lindl.) S. Y. Hu. in Quart. J. Taiwan Mus. 25 (3-4): 213 (1972). Calanthe gracilis* Lindl. (1833). Terrestrial. Fl.: 10. Rare. Distribution: India and Bangladesh (Bandarban and Sylhet). Lindley (1830-40) reported from Sylhet by synonym. Specimen examined: Bandarban: Teracha mukh; 09. 09. 99, M. A. Rahman et al. 5787a (HCU).

15. Cleisostoma subulatum Blume, Bijdr. : 363 (1825). Sarcanthus secundus* Griff. (1851); Sarcanthus subulatus (Blume) Rchb. f. (1857); Saccolabium secundum (Griff.) Ridl. (1907). Reported by its synonym from Sylhet by Hooker (1890b). Epiphytic. Fl.: 8. Rare. Distribution: Bhutan, Cambodia, India, Malaysia, Myanmar, Philippines, Thailand and Bangladesh (Chittagong and Sylhet) No specimen examined.

16. Coelogyne cristata Lindl., Coll. Bot. : t. 33 (1821). Cymbidium speciosissimum Don (1825). Hooker (1890b) reported it from Sylhet. Epiphytic. Fl.: 3-4. Rare. Distribution: Bhutan, India, Nepal and Bangladesh (Cox's Bazar and Sylhet).

17. *Coelogyne punctulata Lindl., Coll. Bot.: sub t. 33 (1821). Cymbidium nitidum* sensu Roxb. (1814). Coelogyne ocellata Lindl. (1830); C. goweri Rchb. f. (1869); C. nitida sensu (Roxb.) Hook. f. (1890b). Reported by its synonym from Sylhet by Roxburgh (1832). Epiphytic. Fl.: 3-4. Specimen deficient. Distribution: Bhutan, India, Myanmar, China, Nepal and Bangladesh (Sylhet).

18. Cymbidium aloifolium (L.) Sw. in Nov. Act. Soc. Upsal. 6: 73 (1799). Epidendrum aloifolium L. (1753); Epidendrum pendulum Roxb. (1795); Cymbidium bicolor Lindl. (1833); Cymbidium erectum Wight (1851). Reported from Chittagong by Heinig (1925). Epiphytic. Fl.: 4-6. Common. Distribution: India, Myanmar to Java, Sri Lanka and Bangladesh (Commonly distributed in Chittagong, Chittagong Hill Tracts, Sylhet and Cox's Bazar). Specimen examined: Habiganj: Rema-Kalenga National Forest, 25. 02. 07, M. Islam 03 (HCU).

19. Dendrobium amoenum Wall in Lindl., Gen. Sp. Orchid. Pl.: 78 (1830). Dendrobium egertoniae Lindl. (1847); D. mesochlorum Lindl. (1847); D. amoena (Wall. ex Lindl.) Kuntze (1891). Hooker (1890b) reported it from Sylhet. Epiphytic. Fl.: 6. Rare. Distribution: India, Myanmar and Bangladesh (Sylhet). Specimen examined: Sylhet: Haripur, 08.07.1981, Moyeen 57 ( $\mathrm{HCU})$.

20. Dendrobium aphyllum (Roxb.) C.E.C. Fischer in Gamble, Fl. Pres. Madras 3: 1416 (1928). Limodorum aphyllum Roxb. (1795); Dendrobium pierardi* Roxb. ex Hook. (1822); D. aphyllum var. cucullatum (Hook. f.) Sarkar (1984). Roxburgh (1832) reported it from Chittagong by synonym. Epiphytic. Fl.: 4-5. Common. Distribution: India, Myanmar and Bangladesh 
(Chittagong, Chittagong Hill Tracts, Cox's Bazar and Sylhet). Specimen examined: Sylhet: Jaintapur; 12. 06. 07, M. Islam and M. K. Huda 12 (HCU).

21. *Dendrobium chryseum Rolfe in Gard. Chron. Ser. 3, 3: 233 (1888). Dendrobium clavatum Wall. ex Lindl. (1852); Callista clavata (Wall. ex Lindl.) Kuntze (1891); Dendrobium tibeticum Schltr. (1921); D. clavatum var. aurantiacum (Rchb. f.) Tang \& Wang (1951). Reported from Sylhet by Hooker (1890b). Epiphytic. Fl.: 6. Specimen deficient. Distribution: Bhutan, China, India, Myanmar, Nepal, Thailand, Vietnam and Bangladesh (Sylhet).

22. Dendrobium fimbriatum Hook., Exot. Fl. : t. 71 (1823). Dendrobium paxtonii Paxt. (1839); D. fimbriatum var. oculatum Hook. f. (1890b); Callista oculata (Hook.) Kuntze (1891). Reported from Chittagong by Prain (1903). Epiphytic. Fl.: 3-5. Rare. Distribution: India and Bangladesh (Chittagong Hill tracts, Cox's Bazar and Sylhet). Specimen examined: Sylhet: Jaintapur, 23.03.86, M. Ahmed 1000 (HCU).

23. Dendrobium formosum Roxb. ex Lindl. in Wall., Pl. Asiat. Rar. 1: 34, t. 29 (1830). Dendrobium formosum Roxb. (1814); D. infundibulum sensu Rchb. f. (1887); Callista formosa (Roxb. ex Lindl.) Kuntze (1891). Reported from Sylhet by Roxburgh (1832). Epiphytic. Fl.: 5. Rare. Distribution: Bhutan, India, Nepal and Bangladesh (Chittagong, Cox's Bazar and Sylhet). Specimens examined: Cox's Bazar: Ukhia, 07.05.84, D.K. Das and M.K. Alam 5015 (HBFRI); Ukia, 08.08.81; Moyeen 63 (HCU).Sylhet: Tamabil, 19.05.1983, Mia 909 (DACB).

24. Dendrobium lindleyi Steud., Nomencl. Bot. ed. 2: 490 (1840). Dendrobium aggregatum* Roxb. (1814); Callista aggregata (Roxb.) Kuntze (1891). Reported by its synonym from Cox's Bazar by Sinclair (1956) and from Sylhet by Uddin et al. (2002). Epiphytic. Fl.: 3-5. Occasional. Distribution: Bhutan, China, India, Laos, Myanmar, Thailand, Vietnam and Bangladesh (Chittagong Hill Tracts, Cox's Bazar and Sylhet). Specimen examined: Rangamati: Sitapahar, Kaptai, 08.01.95, Mezanul Hoque 7344 (HBFRI).

25. *Dendrobium macrostachyum Lindl., Gen. Sp. Orchid. Pl. : 78 (1830). Dendrobium gamblei King \& Pantl. (1897). Reported from Sylhet by Hossain (2002). Epiphytic. Fl.: 5-6. Rare. Distribution: India, Sri Lanka and Bangladesh (Sylhet). Specimen examined: Sylhet: Near Forest School. 22.03.96, Ahmed 96 (HCU).

26. Dendrobium moschatum (Buch-Ham) Sw. in Schltr. Neim. J. Bot. 1: 943 (1805). Dendrobium calceolaria Carey ex Hook. f. (1825-26); Epidendrum moschatum Buch-Ham. (1800). Reported from Chittagong by Prain (1903). Epiphytic. Fl.: 5-7. Rare. Distribution: India and Bangladesh (Chittagong, Chittagong Hill Tracts, Cox's Bazar and Sylhet). Specimen examined: Sylhet: New Forest School; 13.03.56, M. S. Khan Collection number: not available (DUSH).

27. Dendrobium parishii Rchb. f. in Bot. Zeit. 21(31): 237 (1863). Callista parishii (Rchb. f.) Kuntze (1891). Epiphytic. Fl.: 3-5. Scarce. Distribution: India, Malaysia, Myanmar, Thailand and Bangladesh (Bandarban, Rangamati and Sylhet). Specimens examined: Rangamati: Naniarchar, 26.09.1998, M. K. Huda \& S. B. Uddin 469 (HCU); Sylhet: Jaflong; 23.02.1986, M. Ahmed sn (HCU).

28. *Dendrobium pulchellum Roxb ex Lindl., Gen. Sp. Orchid. Pl.: 82 (1830). Dendrobium pulchellum Roxb. (1814); D. dalhausieanum Wall. (1844); Callista pulchella (Roxb. ex Lindl.) Kuntze (1861). Reported from Sylhet by Roxburgh $(1814,1832)$. Epiphytic. Fl.: 2-4. Specimen deficient. Distribution: Bhutan, India and Bangladesh (Sylhet). 
29. *Dendrobium ruckeri Lindl. in Bot. Reg. 29: t. 60, misc. 25, no. 38 (1843). Dendrobium ramosum* sensu Lindl., Gen. \& Sp. Orchid Pl : 82 (1830). Reported from Sylhet by Hooker (1890b). Epiphytic. Fl.: not known. Specimen deficient. Distribution: India and Bangladesh (Sylhet).

30. Didymoplexis pallens Griff., Calcutta J. Nat. Hist. 4: 383, t. 17 (1844). Leucorchis sylvatica Bl. (1849); Arethusa ecristata Griff. (1851); Apetelon minutum Wight (1852); Gastrodia pallens (Griff.) F. Mueller (1870); Didymoplexis brevipes Ohwi. (1937). Reported from Bengal by Hooker (1890b). Saprophytic. Fl.: 4-5. Scarce. Distribution: Afghanistan, Australia, Bhutan, India, Japan, Malaysia to Philippines, New Guinea, Thailand, and Bangladesh (Comilla, Dhaka, Gazipur, Panchagarh and Sylhet by Khanam et al., 2001).

31. Eria pubescens (Hook. f.) Lindl. in Edw., Bot. Reg. 11: t. 904 (1825). Dendrobium pubescens Hook. f. (1890b); Eria flava* Lindl. (1830). Reported by its synonym from Cox's Bazar by Ahmed et al. (1989b). Epiphytic. Fl.: 2-4. Scarce. Distribution: India and Bangladesh (Chittagong and Sylhet). Specimens examined: Sylhet: Jaflong, 22.03.1989, M. Ahmed and Pasha 135 (HCU); Tamabil, 12. 06. 07, M. Islam and M. K. Huda, 04 (HCU).

32. *Erythrodes humilis (B1.) J. J.Smith, Bull, Dep. Agric. Indes Neerl. 13: 11 (1907). Physurus humilis B1., Orch. Archip. Ind. 96: t. 27, f. 2 (1859); Physurus blumei* Lindl. (1840). Reported by its synonym from Sylhet by Lindley $(1830$ - 40). Terrestrial. Fl.: 1-3. Specimen deficient. Distribution: Borneo, India, Java, Sri Lanka and Bangladesh (Sylhet).

33. Gastrochilus calceolaris (Buch-Ham. ex J. E. Smith) D. Don, Prodr. Fl. Nepal.: 32 (1852). Aerides calceolaris Buch.-Ham. ex J. E. Smith (1819); Epidendrum calceolare Buch.-Ham. (1825); Sarcochilus nepalensis Spreng. (1826); Saccolabium calceolare* (Buch.-Ham. ex J. E. Smith) Lindl. (1833); Aerides leopardium Wall. ex Lindl. (1838); A. leopardorum Wall. (1890). Reported by its synonym from Sylhet by Hooker (1890b). Epiphytic. Fl.: 3-5. Specimen deficient. Distribution: Bhutan, China, India, Myanmar, Nepal, Thailand, Vietnam to Malaysia and Bangladesh (Cox's Bazar and Sylhet).

34. *Gastrochilus inconspicuous (Hook. f.) Kuntze, Revis. Gen. Pl. 2: 661 (1891). Saccolabium inconspicuum Hook. f. (1890b); Cymbidium incospicuum Wall. ex Hook. f. (1895); Luisia inconspicua Hook. f. (1898). Reported from Sylhet by Ahmed and Pasha (1998a). Epiphytic. Fl.: 6-7. Rare. Distribution: Bhutan, India, Nepal and Bangladesh (Sylhet). Specimen examined: Sylhet, 05.06.81, M. Ahmed sn (HCU).

35. Geodorum densiflorum (Lam.) Schltr. in Fedde, Repert. 4: 259 (1929). Limodorum densiflorum Lam. (1792); Geodorum dilatatum* R. Br. (1813); Geodorum purpureum R. Br. (1813); Limodorum candidum Roxb. (1814). Reported by its synonym from Sylhet by Roxburgh (1814, 1832). Terrestrial. Fl.: 4. Occasional. Distribution: Australia, Bhutan, China, Fiji, India, Malaysia, Myanmar, New Guinea, Samoa, Solomon Island, Sri Lanka, Tonga and Bangladesh (Chittagong, Chittagong Hill tracts, Gazipur, Mymensigh, Tangail and Sylhet). Specimens examined: Rangamati: Kaptai, 30.08.99, M.A. Rahman et al. 5365 (HCU).

36. Goodyera procera (Wall. ex Ker-Gawl.) Hook. f., Exot. Fl. 1(3): t. 39 (1823). Neottia procera Wall. ex Ker-Gawl. (1822); Goodyera carnea A. Rich. (1841); Epipactis procera (KerGawl.) Eaton (1908). Reported from Sylhet by Lindley (1830) Terrestrial. Fl.: 3-5. Occasional. Distribution: Bhutan, China, India, Japan, Malaysia, Myanmar, Sri Lanka, Taiwan, Philippines and Bangladesh (Chittagong, Cox's Bazar and Sylhet). Specimen examined: Bandarban: Ali Kadam; 30.05.98, M.A. Rahman et al. 2882b (HCU). 
37. Habenaria digitata Lindl. Gen. Sp. Orchid. Pl.: 307 (1835). Habenaria trinervia Wight (1851); H. graveolens Duthie (1906). Hooker (1890b) reported it from Sylhet. Terrestrial. Fl.: 811. Specimen deficient. Distribution: India, Myanmar and Bangladesh (Chittagong and Sylhet).

38. Hetaeria affinis (Griff.) Seidenf. in Oasis, Suppl. 2: 9 (2001). Goodyera affinis Griff. (1851); Cerochilus rubens Lindl. (1854); Rhamphidia rubens (Lindl.) Lindl. (1857); Hetaeria rubens* (Lindl.) Bentham ex Hook. f. (1890). Reported by its synonym from Chittagong by Bruhl (1926). Terrestrial. Fl.: 3-4. Scarce. Distribution: Bhutan, China, India, Myanmar, Thailand, Vietnam and Bangladesh (Chittagong, Mymensingh and Sylhet). Specimen examined: Moulvi Bazar: Lawachara, 27. 02. 07, M. Islam 05 (HCU).

39. Luisia filiformis Hook. f., Fl. Brit. India 6(1): 23 (1890). Luisia grovesi Hook. f. (1890); L. volurcris sensu King \& Pantl. (1898); L. gamblei Durand (1906). Reported from Sylhet by Hooker (1890b). Epiphytic. Fl.: 3-4. Scarce. Distribution: Bhutan, India, Laos, Thailand, Vietnam and Bangladesh (Cox's Bazar and Sylhet). Specimen examined: Sylhet: Jaintapur, 12. 06. 07, M. Islam 13 (HCU).

40. Luisia trichorhiza (Hook. f.) Bl. Rumphia 4: 50 (1849). Vanda trichorhiza Hook. f. (1825); Cymbidium triste sensu Lindl. (1833). Reported from Sylhet by Ahmed and Pasha (1998b). Epiphytic. Fl.: 3-5. Occasional. Distribution: Bhutan, India, Myanmar, Thailand and Bangladesh (Chittagong, Cox's Bazar and Sylhet). Specimens examined: Sylhet: Lama Bazar; 24.03.86, M. Ahmed 102 (HCU); Cox's Bazar: Whykong Reserve Forest; 10.09.99, M.K. Huda et al. 5834 (HCU).

41. Luisia volucris Lindl., Fol. Orchid. 1 (1852). Reported from Sylhet by Hooker (1890b). Epiphytic. Fl.: 3-4. Rare. Distribution: India, Sikkim and Bangladesh (Chittagong and Sylhet).

42. Malaxis acuminata D. Don, Prodr. Fl. Nepal. : 29 (1825). Microstylis wallichii Lindl. (1830); M. biloba Lindl.(1829); Malaxis biloba (Lindl.) Ames (1908); Malaxis wallichii (Lindl.) Deb (1962). Reported from Sylhet by Lindley (1830-40). Terrestrial. Fl.: 7-9. Rare. Distribution: Bhutan, Cambodia, India, Java, Malaysia, Myanmar, Nepal, Philippines, Sumatra, Thailand, Vietnam and Bangladesh (Sylhet and Rangamati). Specimen examined: Rangamati: Kaptai,27.06.98, M.A. Rahman et al. 3229 (HCU).

43. *Malaxis biaurita Lindl., Gen. Sp. Orchid. : 20 (1830). Report from Sylhet by Lindley (1830-40). Terrestrial. Fl.: 7-10. Specimen deficient. Distribution: India and Bangladesh (Sylhet).

44. Micropera rostrata (Roxb.) Balakr. in J. Bombay Nat. Hist. Soc. 67 (1): 66 (1970). Aerides rostrata* Roxb. (1814); Camarotis purpurea Lindl. (1832); Micropera pallida sensu Lindl. (1833); Camarotis pallida (Lindl.) Lindl. (1859); C. rostrata (Roxb.) Roxb. (1864); Sarcochilus purpureus (Lindl.) Benth. ex Hook. f. (1890); Micropera purpurea (Lindl.) Pradhan (1979). Reported by synonym by Roxburgh $(1814,1832)$ from Chittagong and Sylhet. Epiphytic. Fl.: 5-6. Occasional. Distribution: India, Malaysia, Myanmar, Thailand and Bangladesh (Chittagong, Chittagong Hill Tracts and Sylhet).

45. *Nervilia juliana (Roxb.) Schltr. In Bot. Jahrb. Syst. 45: 402 (1911). Arethusa juliana Roxb. (1814); Epipactis juliana Roxb. (1832); Pogonia juliana (Roxb.) Lindl. (1832). Reported from Sylhet by Jayaweera (1981). Terrestrial. Fl.: Not known. Specimen deficient. Distribution: India, Sri Lanka and Bangladesh (Sylhet).

46. *Oberonia mannii Hook. f. Ic. Pl. : t. 2003 (1890). Reported from Sylhet by Hooker (1890a). Epiphytic. Fl.: Not known. Specimen deficient. Distribution: India and Bangladesh (Sylhet). 
47. Oberonia mucronata (D. Don.) Ormerod \& Seidenfaden in Seidenfaden, Contrib. Orch. Flora Thailand XIII: 20 (1997). Stelis mucronata D. Don. (1825); Cymbidium iridifolium* Roxb. (1832); Oberonia iridifolia Lindl., (1830); Malaxis iridifolia (Roxb.) Rchb. f. (1861); Oberonia denticulata var. iridifolia (Roxb.) S. Misra (1989). Reported by its synonym from Sylhet by Roxburgh (1814, 1832). Epiphytic. Fl.: 8-9. Occasional. Distribution: Bhutan, China, India, Indonesia, Malaysia, Nepal, Philippines and Bangladesh (Chittagong and Sylhet). Specimens examined: Sylhet: Lathitila Rain Forest, 29.11.83, M. K. Alam 4742 (HBFRI); Lama Bazar, 21.03.86, M. Ahmed 103 (HCU),: Madhabkunda Eco Park,26. 02. 07, M. Islam 06 (HCU).

48. Oberonia rufilabris Lindl., Sert. Orch. : t. 8 A (1838). Malaxis rufilabris (Lindl.) Rchb. f. (1861). Reported from Sylhet by Hooker (1890a) and from Cox's Bazar by Huda (2000). Epiphytic. Fl.: 8-9. Scarce. Distribution: Bhutan, Cambodia, India, Malaysia, Myanmar, Nepal, Thailand, Vietnam and Bangladesh (Cox's Bazar and Sylhet). Specimen examined: Cox's Bazar: Panerchara Tulabagan; 30.01.99, M.K. Huda et al. 5315 (HCU).

49. Oberonia wallichii Hook. f. Fl. Brit. India. 6: 681 (1890). Oberonia iridifolia Wall. Cat. 1948/2 in part. Reported from Sylhet by Hooker (1890a). Epiphytic. Fl.: Not known. Specimen deficient. Distribution: India and Bangladesh (Cox's Bazar and Sylhet).

50. *Paphiopedilum insigne (Wall. ex Lindl.) Pfitz., in Engler, Bot. Jahrb. 19: 41 (1894). Cypripedium insigne Wall. ex Lindl. (1840). Reported from Sylhet by Lindley (1830-1840). Terrestrial. Fl.: 10-3. Specimen deficient. Distribution: Bhutan, India and Bangladesh (Sylhet).

51. *Paphiopedilum venustum (Wall.) Pfitzer ex Stein, Orchid.- Buch: 489 (1892). Cypripedium venustum Wall. (1820); Cypripedium pardinum Rchb. f. (1869). Reported from Sylhet by Lindley (1830-1840). Terrestrial. Fl.: 3-5. Specimen deficient. Distribution: Bhutan, India and Bangladesh (Sylhet).

52. Papilionanthe teres (Roxb.) Schltr. in Orchis 9: 78 (1915). Dendrobium teres Roxb. (1832); Vanda teres* (Roxb.) Lindl. (1833). Reported by its synonym from Chittagong by Roxburgh (1814, 1832). Epiphytic. Fl.: 3-4. Occasional. Distribution: Bhutan, China, India, Myanmar, Nepal, Thailand, Vietnam and Bangladesh (Chittagong, Chittagong Hill Tracts, Cox's Bazar and Sylhet). Specimens examined: Rangamati: Kaptai, 23.05.83, M. N. Islam sn (HBFRI); Cox's Bazar: Sylhet: Madhabkundu, 16.06.07; M. Islam, 10 (HCU).

53. Pelatantheria insectifera (Rchb. f.) Ridl. in J. Linn. Soc. 32: 373 (1896). Sarcanthus insectifer Rchb. f. (1857). Reported from Chittagong by Hooker (1890b). Epiphytic. Fl.: 9-12. Scarce. Distribution: Bhutan, India, Myanmar, Nepal, Thailand and Bangladesh (Cox's Bazar and Sylhet). Specimen examined: Habiganj: Rema-Kalenga, 25.02.07, M. Islam 07(HCU).

54. Peristylus goodyeroides (D. Don.) Lindl., Gen. Sp. Orchid. : 299 (1835). Habenaria goodyeroides* D. Don (1825). Reported by its synonym from Sylhet by Hooker (1890b). Terrestrial. Fl.: 5-7. Occasional. Distribution: China, India, Indonesia, Malaysia, Philippines and Bangladesh (Chittagong, Rangamati and Sylhet). Specimen examined: Rangamati: Khajachara, 26. 07. 97, M.A. Rahman et al. 1579 (HCU).

55. Phaius tancarvilleae (Banks ex L "Herit) Blume, Mus. Bot. 2: 177 (1856). Limodorum tancanvilleae Banks in L "Herit. (1789); Blettia tanearvilleae Ait (1813); Phajus veratrifolius Wall ex Lindl. (1831); Phaius wallichii* Lindl (1831); Phaius blumei var. assamica Rchb. f. (1882). Reported by its synonym from Sylhet by Hooker (1890b). Terrestrial. Fl.: 2-3. Scarce. Distribution: Australia, Bhutan, China, India, Indonesia, Malaysia, Myanmar, New Guinea, Pacific Islands, Sri Lanka, and Bangladesh (Runctia Forest, Gazni and Sylhet). 
56. *Phalaenopsis taenialis (Lindl.) E.A. Christenson \& Pradhan in Selbyana 9: 168 (1986). Aerides taeniale Lindl. (1833); Doritis taenialis (Lindl.) Hook. f. (1890); Kingiella taenialis (Lindl.) Rolfe (1917); Kingidium taenialis* (Lindl.) P.F. Hunt (1970). Reported by its synonym from Sylhet by Ahmed et al. (1992). Epiphytic. Fl.: 5. Rare. Distribution: Bhutan, China, India, Nepal and Bangladesh (Sylhet). Specimen examined: Sylhet: Jainta Bazar, 24.03.86, M. Ahmed sn (HCU).

57. Pholidota imbricata Hook. f., exot. Fl. 2: t. 138 (1825). Cymbidium imbricatum* Roxb. (1832); Coelogyne imbricata (Roxb.) Rchb. f. (1861); Pholidota asamica Regel. (1890); Pholidota pallida sensu Holtum (1964). Reported by its synonym from Chittagong and Sylhet by Roxburgh (1814, 1832). Epiphytic. Fl.: 6-7. Occasional. Distribution: Australia, China, India, Laos, Malaysia, Myanmar, Nepal, Nicobar Island, New Guinea, Pacific Islands, Sri Lanka, Thailand and Bangladesh (Chittagong, Chittagong Hill Tracts, Cox's Bazar and Sylhet). Specimen examined: Cox's Bazar: Whykong Reserve Forest, 11.09.99, M.K. Huda et al. 5848 (HCU).

58. *Podochilus khasianus Hook. f., Fl. Brit. India 6: 81 (1890). Podochilus chinensis Schltr. (1924). Reported from Sylhet by Hooker (1890b). Epiphytic. Fl.: 3-5. Specimen deficient. Distribution: Bhutan, China, India and Bangladesh (Sylhet).

59. Pomatocalpa decipiens (Lindl.) J. J. Smith, Natuurk. Tijdscr. Ned. Indie 72: 33 (1912). Cleisostoma decipiens Lindl. (1844); Saccolabium decipiens (Lindl.) Alston (1931). Reported from Habiganj by Uddin et al. (1999). Epiphytic. Fl.: 3. Scarce. Distribution: Sri Lanka and Bangladesh (Sylhet). Specimen examined: Habiganj: Chunarughat, Rema Kalenga Wild life Sanctuary, Kalega beat, Habiganj: 18.03.99 (DACB and DUSH).

60. Rhynchostylis retusa (L.) Blume, Bijdr. : 286, t. 49 (1825). Epidendrum retusum L. (1753); Aerides guttatum* Roxb. (1832); Saccolabium rheedii Wight (1851); Saccolabium guttatum Lindl. (1833); Saccolabium berkeleyi Rchb. f. (1883). Reported by its synonym from Dhaka by Roxburgh (1814, 1832). Epiphytic. Fl.: 5-7. Common. Distribution: Bhutan, India, Malaysia, Myanmar, Nepal, Philippines, Sri Lanka and Bangladesh (throughout Bangladesh). Specimen examined: Sylhet: Shreepur, 12. 06. 07, M. Islam 08 (HCU).

61. Robiquetia spathulata (B1.) J. J. Sm. in Nat. Tijdschr. Ned. Ind. 72 : 114 (1912). Cleisostoma spathulatum Bl. (1825); Saccolabium densiflorum Lindl. (1832); Cleisostoma spicatum Lindl. (1847). Reported from Sylhet by Seidenfaden (1988). Epiphytic. Fl.: 5-7. Scarce. Distribution: China, India, Indo-china, Indonesia, Malaysia, Myanmar, Singapore, Thailand and Bangladesh (Cox's Bazar and Sylhet).

62. Robiquetia succisa (Lindl.) Seid. \& Garay in Bot. Tidsskr. 67: 119 ( 1972). Sarcanthus succisus Lindl. (1826); Oecoclades paniculata Lindl.(1833); Saccolabium parvulum Lindl. (1859); S. buccosum* Rchb. f. (1871); Robiquetia paniculata (Lindl.) J. J. Smith (1912); Sarcanthus henryi Schltr. (1919). Reported from Sylhet by Hooker (1890b). Epiphytic. Fl.: 6-7. Scarce. Distribution: Bhutan, Cambodia, China, India, Laos, Myanmar, Thailand, Vietnam and Bangladesh (Chittagong, Chittagong Hill Tracts and Sylhet).

63. Saccolabiopsis pusilla (Lindl.) Seidenf. \& Garay in Bot. Tidsskr. 67: 118, f. 33 (1972). Saccolabium pusillum Lindl. (1858); Saccolabium pumilio* Rchb. f. (1890). Reported by its synonym from Sylhet by Hooker (1890b). Epiphytic. Fl.: 4-6. Scarce. Distribution: Bhutan, India, Myanmar and Bangladesh (Rangamati and Sylhet). Specimen examined: Chittagong: Gondamara, Dhoplachari, Chandanaish, 24.07.99, M.K. Huda et al. 5152 (HCU). 
64. *Saccolabium cephalotes Hook. f., Fl. Brit. India 5: 63 (1890). Acampe cephalotes Lindl. Reported from Sylhet by Hooker (1890b). Epiphytic. Fl.: Not known. Specimen deficient. Distribution: India and Bangladesh (Sylhet).

65. *Schoenorchis gemmata (Lindl.) J. J. Smith in Natuurk. Tijdschr. Ned.-Indie. 72: 100 (1912). Saccolabium gemmata Lindl. (1838); S. geminatum* (Lindl.) Hook. f.(1890); Cleisostoma gemmatum (Lindl.) King \& Pantl. (1898). Reported by its synonym from Jyantia (Sylhet) by Hooker (1890b). Epiphytic. Fl.: 5-6. Specimen deficient. Distribution: Bhutan, Cambodia, China, India, Laos, Myanmar, Thailand, Vietnam and Bangladesh (Sylhet).

66. Smitinandia micrantha (Lindl.) Holttum in Gard. Bull. Singapore, 25: 106 (1969). Saccolabium micranthum* Lindl. (1833); Cleisostoma micranthum (Lindl.) King \& Pantl. (1898). Reported by its synonym from Sylhet by Hooker (1890b). Epiphytic. Fl.: 7-9. Scarce. Distribution: Bhutan, India, Laos, Malaysia, Myanmar, Nepal, Thailand, Vietnam and Bangladesh (Cox's Bazar and Sylhet).

67. *Spathoglottis pubescens Lindl., Gen. Sp. Orchid. : 120 (1831). Spathoglottis pubescens var. parviflora (Lindl.) Hook. f. (1890). Collected from East Bengal by Griffith, CAL- 5194 (Huda et al., 1999) and reported from Sylhet by Lindley (1830 - 40). Terrestrial. Fl.: 6-9. Specimen deficient. Distribution: Bhutan, China, India, Myanmar and Bangladesh (Sylhet).

68. Staurochilus ramosus (Lindl.) Seidenf., in Opera Bot. 95 : 95 (1988). Saccolabium ramosum Lindl. (1833); Aerides ramosum Wall. (1833); Cleisostoma ramosum* (Lindl.) Hook. f. (1890); Gastrochilus ramosus (Lindl.) Kuntze (1891); Sarcanthus ramosus (Lindl.) J. J. Smith (1912); Pomatocalpa ramosum (Lindl.) Summerh. (1948). Reported by its synonym from Sundarbans by Hooker (1888-90) and from Cox's Bazar by Seidenfaden (1988). Epiphytic. Fl.: 5. Common. Distribution: Bhutan, India, Myanmar, Thailand and Bangladesh (Chittagong, Chittagong Hill Tracts, Cox's Bazar and Sylhet). Specimen examined: Habiganj: Rema-Kalenga, 25. 02. 07, M. Islam 09 (HCU).

69. *Tainia latifolia (Lindl.) Rchb. f. in Bonplandia 5: 54 (1857). Ania latifolia Lindl. (1831); Mitopetalum latifolium (Lindl.) Bl. (1856); Eulophia hastate Lindl. (1859); Tainia hastata (Lindl.) Hooker (1890); T. khasiana Hook. f.(1890). Reported from Sylhet by Hook. f. (1890a). Terrestrial. Fl.: 9-11. Specimen deficient. Distribution: Bhutan, China, India, Laos, Myanmar, Thailand, Vietnam and Bangladesh (Sylhet).

70. *Tropidia angulosa (Lindl.) Bl., Coll. Orchid. : 122 (1859). Decasinea angulosa Wall. (1832); Cnemidia angulosa Lindl. (1840); Govindova nervosa Wight (1853); Tropidia govindovii Bl. (1858). Reported from Sylhet by Hooker (1890b). Terrestrial. Fl.: 8-11. Rare. Distribution: Bhutan, China, India, Malaysia, Myanmar, Sumatra, Thailand, and Bangladesh (Sylhet).

71. Tropidia curculigoides Lindl., Gen. Sp. Orchid. Pl. : 497 (1840). Tropidia squamata Bl. (1859); T. assamica Bl. (1858); T. graminea Bl. (1859); T. formosana Rolfe (1895). Report from Sylhet by Hooker (1890b). Terrestrial. Fl.: 9-12 . Occasional. Distribution: Bhutan, China, India, Malaysia, Myanmar and Bangladesh (Cox's Bazar and Sylhet). Specimen examined: Rangamati: Kaptai, Sitapahar, 03.09.99, M.A. Rahman et al. 5591 (HCU).

72. *Vanda crisata Lindl., Gen. Sp. Orchid. Pl. : 216 (1833). Aerides cristatum Wall. (1832); Trudelia cristata (Lindl.) Senghas (1988). Reported from Sylhet by Hooker (1890b). Epiphytic. Fl.: 5. Rare. Distribution: Bhutan, India, Nepal and Bangladesh (Sylhet). 
73. Vanilla parishii Rchb. f., Otia Bot. Hamb. : 39 (1878). Reported from Rangamati by Prain (1903). Hemi-epiphytic. Fl.: Not known. Scarce. Distribution: India, Myanmar and Bangladesh (Chittagong, Cox's Bazar and Sylhet).

74. *Zeuxine flava (Wall. ex Lindl.) Trimen, Syst. Cat. Fl. Pl. : 90 (1885). Etaeria flava Lindl. (1832); Monochilus flavum Wall. ex Lindl. (1840). Reported from Sylhet by Seidenfaden (1978). Terrestrial. Fl.: 5. Rare. Distribution: Bhutan, India, Nepal, Thailand and Bangladesh (Sylhet). Specimen examined: Sylhet: Tamabil; 11.03.56, M. S. Khan (DUSH).

75. Zeuxine nervosa (Wall. ex Lindl.) Bentham ex C.B. Clarke in J. Linn. Soc., Bot. 25: 73 (1889). Monochilus nervosum Wall. ex Lindl. (1840); Haplochilus nervosum (Wall. ex Lindl.) D. Dietrich (1852). Reported from Comilla and Sylhet by Hooker (1890b). Terrestrial. Fl.: 3-4. Scarce. Distribution: Bhutan, China, India, Philippines, Taiwan, and Bangladesh (Comilla, Mymensingh and Sylhet). Specimen examined: Mymensingh; 04.03.77, M. Rahman (DACB).

\section{Discussion}

A total of 75 orchid species were recorded from greater Sylhet, out of which 48 species are epiphytic, 25 species are terrestrial, one is saprophytic (Didymoplexis pallens Griff.) and another one is hemiepiphytic (Vanilla parishii Rchb. f.). Based on literature and the present field work, 26 species were found to occur in Sylhet region only. Thirty-seven out of 49 genera are monotypic indicating a narrower diversity of the family in the studied region. Herbarium specimens of 53 orchid species are available in different herbaria of Bangladesh, namely DACB, DUSH, HCU, HBFRI and HBCSIR. Only 17 orchid species were found in the present survey and are housed at HCU. 22 orchid species were not found in the last 50 years from greater Sylhet region or other parts of Bangladesh.

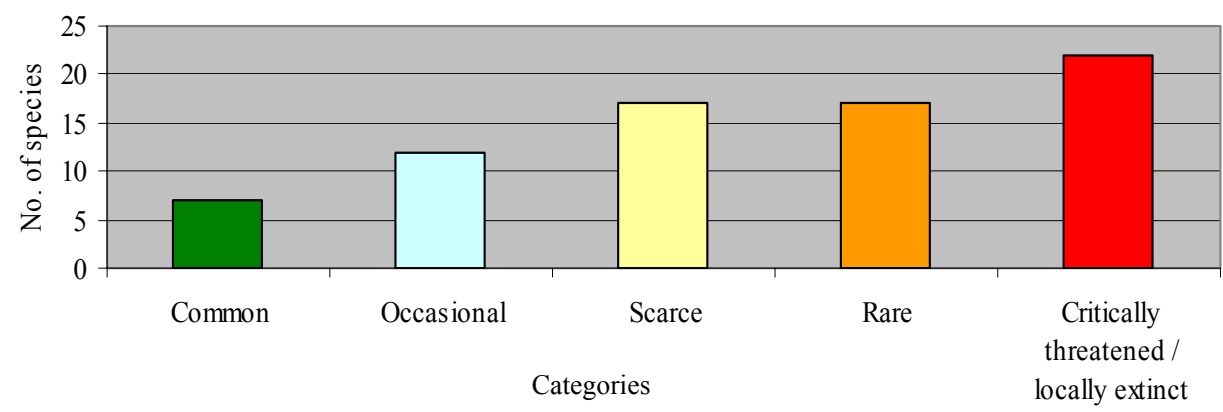

Fig. 1. Abundance status of the orchid species distributed in Sylhet.

Orchids are under great threats to their existence in the natural habitats due to biotic pressures, like illegal felling of large host trees, clearing off forest floor, forest fire and collection of horticultural valuable orchid species. Abundance status based on the availability of different orchid species in the natural habitat of the studied area and previous reports is presented in Fig. 1. It indicates that $30 \%$ orchid species in the Sylhet region have no data of their present occurrence and probably locally extinct from this area or critically threatened. So, both in-situ and ex-situ conservation measures along with public awareness programmes need to be undertaken. 


\section{References}

Abraham, A and Vatsala, P. 1981. Introduction to Orchids. Tropical Botanic Garden and Research Institute, Trivandram, India, $533 \mathrm{pp}$.

Ahmed, M. and Pasha, M.K. 1993. A taxonomic account of Sarcanthus Lindl. (Orchidaceae) from Bangladesh. J. Econ. Tax. Bot. 17(2): 487-491.

Ahmed, M. and Pasha, M.K. 1993a. A taxonomic account of Thrixspermum Lour. (Orchidaceae) from Bangladesh. J. Asiat. Soc. Bang. Sci. 19(1): 35-42.

Ahmed, M. and Pasha, M.K. 1994. A taxonomic account of Hetaeria Bl. (Orchidaceae) from Bangladesh. Chittagong Univ. Stud. Part II: Sci. 18(2): 179-182.

Ahmed, M. and Pasha, M.K. 1998. A taxonomic account of Eulophia R. Br. (Orchidaceae) from Bangladesh. J. Asiat. Soc. Bang. Sci. 24(1): 11-22.

Ahmed, M. and Pasha, M.K. 1998a. Gastrochilus inconspicuum (Hooker, f.) Seidenf. (Orchidaceae) - A new angiospermic record for Bangladesh. Bangladesh J. Bot. 27(2): 143-146.

Ahmed, M. and Pasha, M.K. 1998b. A taxonomic account of Luisia Gaud. (Orchidaceae) from Bangladesh. J. Bom. Nat. Hist. Soc. 95: 301-306.

Ahmed, M. and Pasha, M.K. 1999. A taxonomic account of Robiquetia Gaud. (Orchidaceae) from Bangladesh. J. Bom. Nat. Hist. Soc. 96(3): 499-502.

Ahmed, M., Pasha, M.K. and Khan, M.A.A. 1989. A taxonomic account of Aerides Lour. (Orchidaceae) from Bangladesh. Bangladesh J. Bot. 18(2): 147-155.

Ahmed, M., Pasha, M.K. and Khan, M.A.A. 1989a. Agrostophyllum khasianum Griff. - A new record for Bangladesh. Bangladesh J. Bot. 18(1): 101-104.

Ahmed, M., Pasha, M.K. and Khan, M.A.A. 1989b. Eria flava Lindl. (Orchidaceae) - A new record for Bangladesh. Bangladesh J. Bot. 18(2): 223-226.

Ahmed, M., Pasha, M.K. and Khan, M.A.A. 1992. Kingidium taenialis (Lindl.) P. F. Hunt (Orchidaceae) - A new record for Bangladesh. Bangladesh J. Bot. 21(2): 283-285.

Bruhl, P. 1926. A guide to the orchids of Sikkim. Calcutta and Simla, Thacker, Spink \& Co. 208 pp.

Chowdhery, H.J. 1998. Orchid flora of Arunachal Pradesh. Bishen Singh Mahendra Pal Singh, Dehra Dun, India, $824 \mathrm{pp}$.

Heinig, R.L. 1925. List of plants of Chittagong collectorate and Hill Tracts. Darjeeling, pp. 68-70.

Hooker, J.D. 1890a. Flora of British India (Orchideae), Reeve \& Co., Kent, England. 5: 667-864.

Hooker, J.D. 1890b. Flora of British India (Orchideae), Reeve \& Co., Kent, England. 6: 1-224.

Hossain, A.B.M.E. 2002. A taxonomic report on the genus Dendrobium Sw. (Orchidaceae) from Bangladesh. Bangladesh J. Plant Taxon. 9(2): 47-55.

Huda, M. K., Rahman, M.A. and Wilcock, C.C. 1999. A preliminary checklist of orchid taxa occurring in Bangladesh. Bangladesh J. Plant Taxon. 6: 69-85.

Huda, M. K. 2000. Diversity, Ecology, Reproductive Biology and Conservation of orchids of South East Bangladesh. Doctoral thesis. University of Aberdeen. UK. 266 pp. (Unpublished).

Huda, M.K. Rahman, M.A. and Wilcock, C.C. 2001. Notes on the Orchidaceae of Bangladesh-1 : Some new records. Bangladesh J. Plant Taxon. 8(2): 9-17.

Huda, M.K. 2008. An up to date enumeration of the family Orchidaceae from Bangladesh. J. Orchid Soc. India 21(1-2): 35-49.

Huda, M.K. 2008a. Orchidaceae In: Ahmed, Z.U., Hassan, M.A., Begum, Z.N.T., Khondker, M., Kabir, S.M.H., Ahmad, M., Ahmed, A.T.A., Rahman, A.K.A. and Haque, E.U. (Eds), Encyclopedia of Flora and Fauna of Bangladesh. Vol. 12. Angiosperms: Monocotyledons (Orchidaceae- Zingiberaceae). Asiatic Society of Bangladesh, Dhaka, pp. 1-148.

Jayaweera, D.M.A. 1981. Orchidaceae. In: Dassanayakake, M.D. and Fosberg, F.R. (Eds), Flora of Ceylon, Vol. 2. Amerind Publishing Co. Ltd. New Delhi, pp. 1-386.

Joseph, J. 1987. Orchids of Nilgiris. Botanical Survey of India, Govt. Press of India, 186 pp. 
Khanam, M., Uddin, M.Z., Khan, M.S. and Hassan, M.A. 2001. Our present knowledge on the terrestrial orchidaceous taxa from Bangladesh. Bangladesh J. Plant Taxon. 8(2): 35-49.

Khan, M.S. and Halim, M. 1987. Bulbophyllum lilacinum Ridley - A new angiospermic record for Bangladesh. Bangladesh J. Bot. 16(2): 203-205.

Lindley, J. 1830-40. The genera and species of orchidaceous plants. Indian reprint 1983. Bishen Singh Mahendra Pal Singh, Dehra Dun, India.

Misra, S. 2000. Untamed Orissa- Wild Orissa, Bhubanswar, Orissa in Anonymous (Ed.) pp-25-30.

Pasha, M.K. 1984. Taxonomic studies of orchids of Chittagong division, Project report. Sponsored by the University Grant Commission, Dhaka, Bangladesh.

Prain, D. 1903. Bengal Plants, Vol. 2. Calcutta, pp. 750-777.

Rao, T.A. 1998. Conservation of wild orchids of Kodagu in the Western Ghats, India, pp. 192-230.

Roxburgh, W. 1814 (reprint 1980). Hortus Benghalensis. Boerhavve Press, Leiden.

Roxburgh, W. 1832. Flora Indica (Gynadria Monandria). ed.2,2: 609-622.

Seidenfaden, G. 1978. Orchid genera in Thailand 6. Neottoideae. Dansk Bot. Ark. 32: 1-195.

Seidenfaden, G. 1988. Orchid genera in Thailand XIV. fifty nine Vandoid genera. Opera Bot. 95: 1-398.

Sinclair, J. 1956. The Flora of Cox's Bazar, East Pakistan. Bull. Bot. Soc. Beng. 9(2): 107-108.

Uddin, M.Z., Khan, M.S., Hassan, M.A. and Khanam, M. 1999. Pomatocalpa decipiens (Lindl.) J. J. Smith A new orchid record for Bangladesh. Bangladesh J. Bot. 28(2): 169-171.

Uddin, M.Z., Khan, M.S., Hassan, M.A. and Khanam, M. 2000. Brachycorythis helferi (Rchb. f.) Summerh. A new orchid record for Bangladesh. Bang. J. Plant Taxon. 7(1): 73-75.

Uddin, M.Z., Khan, M.S. and Hassan, M.A. 2002. An annotated checklist of angiospermic flora of RemaKalenga Wildlife Sanctuary (Habiganj) in Bangladesh -I. Liliopsida (Monocots). Bangladesh J. Plant Taxon. 9(2): 57-66. 